
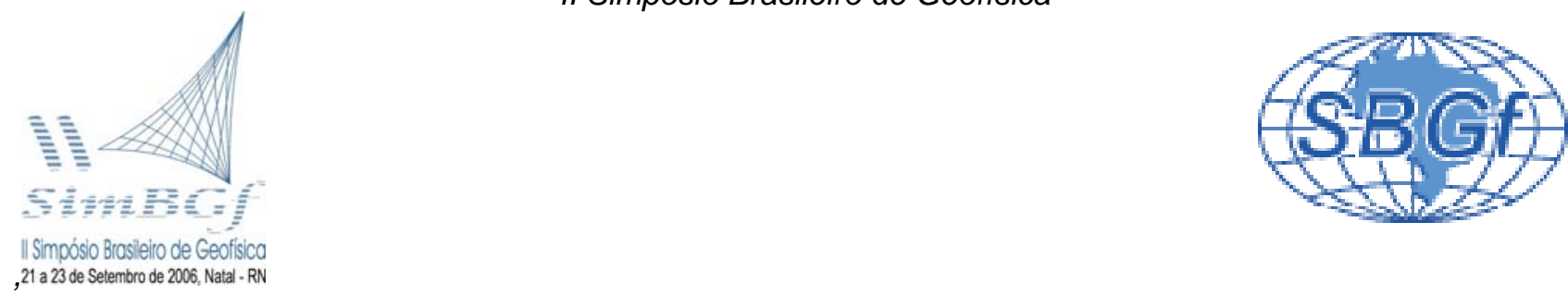

\title{
Resistividade 2D - Profundidade de Investigação e Resolução do Objetivo
}

\author{
Otávio Coaracy Brasil Gandolfo ${ }^{1,2}$ \\ José Domingos Faraco Gallas ${ }^{2}$ \\ ${ }^{1}$ Instituto de Pesquisas Tecnológicas do Estado de São Paulo - IPT \\ ${ }^{2}$ Instituto de Geociências da Universidade de São Paulo - USP
}

\section{Resumo}

Este trabalho apresenta os resultados de um levantamento de eletrorresistividade mostrando como podem ser alcançados, de forma simultânea, dois objetivos em uma aquisição de dados geofísicos: resolução e profundidade de penetração.

Os dados de caminhamentos elétricos foram adquiridos com o arranjo dipolo-dipolo, utilizando o recurso de executar, no mesmo perfil do levantamento, aquisição com distintos espaçamentos entre dipolos.

Os resultados são apresentados sob a forma de pseudo-seções modificadas e modelos 2D gerados por programa de inversão de dados. Com esta modalidade de aquisição foi possível alcançar maiores profundidades de investigação sem a perda de resolução nas profundidades mais rasas.

Informações de uma sondagem mecânica e uma Sondagem Elétrica Vertical (SEV) realizada sobre o perfil possibilitaram checar a validade do modelo $2 \mathrm{D}$ resultante.

\section{Introdução}

Resolução e profundidade de penetração são termos corriqueiros utilizados pela comunidade geofísica. Estes parâmetros guardam entre si uma relação geralmente inversa, ou seja, com maiores profundidades de investigação perde-se resolução e, de forma contrária, maiores resoluções podem ser alcançadas ao custo de uma penetração limitada.

Atingir maiores profundidades sem, entretanto, perder resolução nas porções mais rasas da investigação representa um objetivo nem sempre alcançado nos levantamentos e constitui um dilema por vezes crucial, particularmente nos caminhamentos elétricos de eletrorresistividade.

Especificando o caso do arranjo dipolo-dipolo, mais comumente empregado para o caminhamento elétrico, sabe-se que a profundidade de investigação é função, além das relações entre as resistividades, do espaçamento $\underline{\boldsymbol{a}}$ entre dipolos $(\mathbf{a}=\mathrm{AB}=\mathrm{MN})$. Para investigação de detalhes, faz-se necessária a utilização de dipolos com pequenos espaçamentos, o que limita a profundidade de investigação.
O recurso de se utilizar, em um mesmo perfil de levantamento, vários espaçamentos entre dipolos atende a estas duas necessidades, resolução e profundidade de penetração, como será demonstrado neste trabalho.

\section{Levantamento de Campo}

No levantamento de campo, foi estabelecido um perfil de 40 metros de extensão sobre o qual foram realizados os caminhamentos elétricos utilizando o arranjo dipolo-dipolo com quatro distintos espaçamentos entre dipolos: $4 \mathrm{~m}, 2 \mathrm{~m}, 1 \mathrm{~m}$ e $0,5 \mathrm{~m}$. Foi também realizada uma SEV na estaca $12 \mathrm{~m}$ do perfil do levantamento.

O equipamento utilizado para efetuar as medidas de campo foi um ABEM SAS-300 de um canal. A Tabela 1 sintetiza os parâmetros referentes aos levantamentos executados.

Tabela 1 - Parâmetros dos caminhamentos elétricos utilizando o arranjo dipolo-dipolo.

\begin{tabular}{|c|c|c|}
\hline $\begin{array}{c}\text { Espaçamento } \\
\text { entre dipolos } \\
(\mathbf{a}=\mathrm{AB}=\mathrm{MN})\end{array}$ & $\begin{array}{c}\text { Níveis de } \\
\text { investigação }\end{array}$ & $\begin{array}{c}\text { Número } \\
\text { de medidas } \\
\text { (pontos na } \\
\text { pseudo-seção) }\end{array}$ \\
\hline $0,5 \mathrm{~m}$ & 12 & 870 \\
\hline $1 \mathrm{~m}$ & 12 & 390 \\
\hline $2 \mathrm{~m}$ & 10 & 135 \\
\hline $4 \mathrm{~m}$ & 6 & 33 \\
\hline
\end{tabular}

Os ensaios foram realizados nas proximidades da Raia Olímpica da USP. Neste local há disponibilidade de furos de sondagens (executados para instalação de poços de monitoramento) e outros dados diretos (trincheira) a partir dos quais foram obtidas informações como o perfil litológico, profundidade do nível d'água e teores de umidade do solo (Paixão, 2005).

\section{Geologia da área}

A área encontra-se sobre a Bacia Sedimentar de São Paulo, onde afloram as Formações São Paulo (Grupo Taubaté) e Itaquaquecetuba.

A Tabela 2 mostra o perfil litológico da área estudada, obtido de uma sondagem localizada nas proximidades da estaca $12 \mathrm{~m}$ do perfil geofísico de $40 \mathrm{~m}$, coincidente com o local onde foi realizada a SEV. 
Tabela 2 - Perfil litológico segundo informações de sondagem localizada nas proximidades da estaca $12 \mathrm{~m}$

\begin{tabular}{|c|c|}
\hline Descrição da litologia & Profundidade \\
\hline $\begin{array}{c}\text { Solo com matéria orgânica, argila } \\
\text { e areia fina a média }\end{array}$ & $0-0,2 \mathrm{~m}$ \\
\hline $\begin{array}{c}\text { Areia, silte e argila com presença de } \\
\text { seixos }\end{array}$ & $0,2 \mathrm{~m}-0,7 \mathrm{~m}$ \\
\hline Argila siltosa inconsolidada & $0,7 \mathrm{~m}-1,6 \mathrm{~m}$ \\
\hline Argila muito plástica & $1,6 \mathrm{~m}-3,3 \mathrm{~m}$ \\
\hline $\begin{array}{c}\text { Areia fina com argila, gradando para } \\
\text { frações mais grossas na base }\end{array}$ & $>3,3 \mathrm{~m}$ \\
\hline
\end{tabular}

As três primeiras litologias descritas na Tabela 2 (da superfície até $1,6 \mathrm{~m}$ de profundidade) apresentam pouco conteúdo de água em sua formação estando, portanto, relativamente secas.

Abaixo destas encontra-se uma camada de argila plástica, bastante homogênea e que contêm alto teor de umidade.

Este teor volta a diminuir na camada de areia fina, situada abaixo da argila plástica, cujo topo se encontra a 3,3m de profundidade.

\section{Resultados}

\subsection{Modelo geoelétrico 1D}

A Figura 1 apresenta a curva de resistividade aparente da SEV realizada na estaca $12 \mathrm{~m}$ e o respectivo modelo geoelétrico interpretado. A variação da resistividade em profundidade é dada pela seguinte distribuição: $\rho_{1}>\rho_{2}<\rho_{3}>\rho_{4}$.

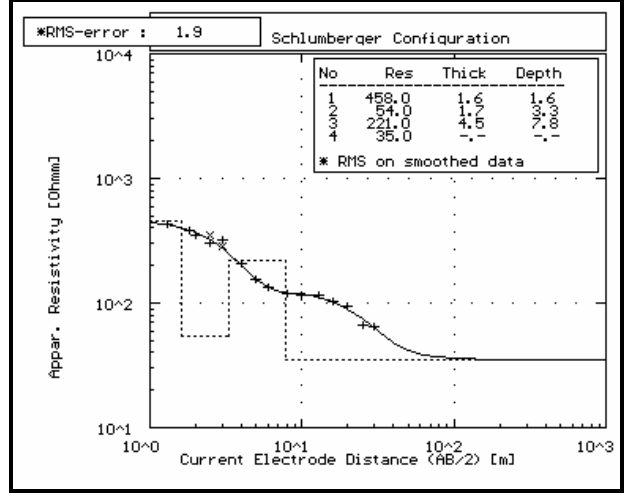

Figura 1 - SEV realizada na estaca $12 \mathrm{~m}$ do perfil de caminhamento.

As seções 2D que serão apresentadas neste trabalho conseguiram imagear de forma evidente as três primeiras camadas deste modelo geoelétrico (resistividades iguais a $\rho_{1}, \rho_{2}$ e $\rho_{3}$ ).

O mesmo padrão de distribuição vertical de resistividade obtido pela SEV $\left(\rho_{1}>\rho_{2}<\rho_{3}\right)$, foi também verificado nas seções, como será visto a seguir.

A SEV conseguiu ainda identificar uma quarta camada geoelétrica (resistividade igual a $\rho_{4}$ ), mais profunda, a aproximadamente $8 \mathrm{~m}$, onde a resistividade volta a cair. Esta camada, por sua vez, não pode ser visualizada nas seções 2D.

\subsection{Pseudo-seções de resistividade aparente}

A Figura 2 apresenta as pseudo-seções correspondentes aos levantamentos com espaçamento entre dipolos igual a $0,5 \mathrm{~m}$ (a), $1 \mathrm{~m}$ (b), $2 \mathrm{~m}$ (c) e $4 \mathrm{~m}$ (d) plotadas de acordo com a representação proposta por Hallof (1957).

Edwards (1977) observou que dados coletados sob um mesmo perfil e com diferentes espaçamentos entre os dipolos, podem ser combinados em uma única pseudo-seção (denominada pelo autor de "pseudo-seção modificada"), se plotados de acordo com os coeficientes empíricos por ele determinados.

Este procedimento possibilita um aumento da razão sinal-ruído nos dados, acarretando uma "sobreposição de medidas", além da possibilidade de serem alcançadas maiores profundidades de investigação sem, entretanto, causar a perda de resolução nas porções mais rasas em subsuperfície.

Na Figura 3 encontra-se ilustrada a plotagem de dados correspondente a três distintos espaçamentos em uma pseudo-seção modificada. Observa-se a perda de resolução com a profundidade e, nas porções mais rasas, uma maior densidade de dados (conseqüentemente, uma melhor definição e detalhamento).

Seguindo este procedimento, os dados adquiridos com os diferentes espaçamentos (Figura 2) foram agrupados, gerando a pseudo-seção modificada que é apresentada na Figura 4.

\subsection{Seções modeladas}

Para inversão dos dados foi utilizado o programa RES2DINV (Geotomo Software, 2004).

As seções 2D modeladas, com espaçamentos 0,5m, 1m e 2m, são apresentadas nas Figuras 5, 6 e 7, respectivamente.

As tonalidades em azul representam os valores altos de resistividade enquanto os tons avermelhados, as baixas resistividades elétricas.

Com o espaçamento de $0,5 m$ (Figura 5) foi identificado apenas o estrato geoelétrico superior de alta resistividade $\left(\rho_{1}\right)$. A aquisição com o dipolo de $1 \mathrm{~m}$ (Figura 6) já permitiu a visualização da segunda camada, onde as resistividades caem de forma acentuada $\left(\rho_{2}\right)$. O imageamento da terceira camada geoelétrica, onde os valores de resistividade voltam a subir $\left(\rho_{3}\right)$, foi possível apenas com a utilização do dipolo de $2 \mathrm{~m}$ (Figura 7).

O software RES2DINV apresenta um recurso bastante interessante que é a possibilidade de se utilizar no processo de inversão, múltiplos espaçamentos entre dipolos, tal como apresentado na pseudo-seção modificada da Figura 4. A seção final modelada, contendo os dados dos quatro espaçamentos utilizados $(0,5 \mathrm{~m}, 1 \mathrm{~m}, 2 \mathrm{~m}$ e $4 \mathrm{~m})$, é apresentada na Figura 8.

A seção final mostrou grande coerência com as demais seções apresentadas de forma individual para cada espaçamento. Foi também verificada uma boa correlação com o modelo geoelétrico fornecido pela SEV, o qual está lançado sobre a seção no ponto onde a mesma foi realizada (estaca 12m).

A camada superior em azul corresponde a porção seca do solo (litologias presentes da superfície 
até $1,6 \mathrm{~m}$ de profundidade), heterogênea e com elevados valores de resistividade elétrica $\left(\rho_{1}\right)$

A camada de argila plástica, representada em vermelho nas seções modeladas, retém muita umidade e, desta forma, apresenta valores baixos de resistividade $\left(\rho_{2}\right)$.

Abaixo da argila plástica, as resistividades voltam a subir na camada de areia fina (resistividade igual a $\rho_{3}$ ), cujo topo se encontra a aproximadamente $3,3 m$ de profundidade.

\section{Conclusões}

Os resultados apresentados neste trabalho mostraram os excelentes resultados obtidos de uma aquisição de eletrorresistividade utilizando o arranjo dipolo-dipolo com múltiplos espaçamentos entre eletrodos sobre o mesmo perfil e apresentação conjunta destes dados em uma mesma pseudo-seção/seção modelada.

As seções mostraram de forma clara que o detalhe obtido nos níveis mais superficiais apresenta um elevado grau de resolução, quase sempre o objetivo principal de uma prospecção muito rasa, onde o detalhamento preciso é exigido. Por outro lado, não houve perda de penetração, uma vez que nestas mesmas seções também podem ser observadas informações relativas aos níveis mais profundos, casos em que a resolução detalhada normalmente não é crucial.

Desta forma, conclui-se que esta metodologia permite conciliar alcançar maiores profundidades sem a perda de resolução nas porções mais rasas.

Isto é confirmado comparando-se a seção 2D de eletrorresistividade, resultante da inversão dos dados, que mostrou uma evidente correlação com o modelo geoelétrico 1D obtido através da SEV e também com as informações diretas de geologia obtidas pela sondagem.

\section{Agradecimentos}

À FAPESP, pelo apoio concedido no Projeto de Auxílio à Pesquisa (Processo 04/01239-8) que possibilitou a execução deste trabalho.

\section{Referências}

EDWARDS, L. S. 1977. A modified pseudosection for resistivity and induced-polarization. Geophysics, 42: 1020-1036.

GEOTOMO SOFTWARE. 2004. RES2DINV (v.3.54) and RES3DINV (v.2.14) for Windows 98/ME/2000/NT/XP. Geoelectrical Imaging 2D \& 3D.

HALLOF, P. G. 1957. On the interpretation of resistivity and induced polarization measurements: Cambridge, MIT, Ph. D. thesis.
PAIXÃO, M. S. G. 2005. Análise da acurácia das estimativas de posicionamento do nível freático e dos teores de umidade do solo com o emprego dos métodos de sísmica de refração rasa e georadar a partir de um estudo no Campus da USP, São Paulo/SP. 117p. Dissertação (Mestrado), Instituto de Astronomia, Geofísica e Ciências Atmosféricas -IAG, USP. 


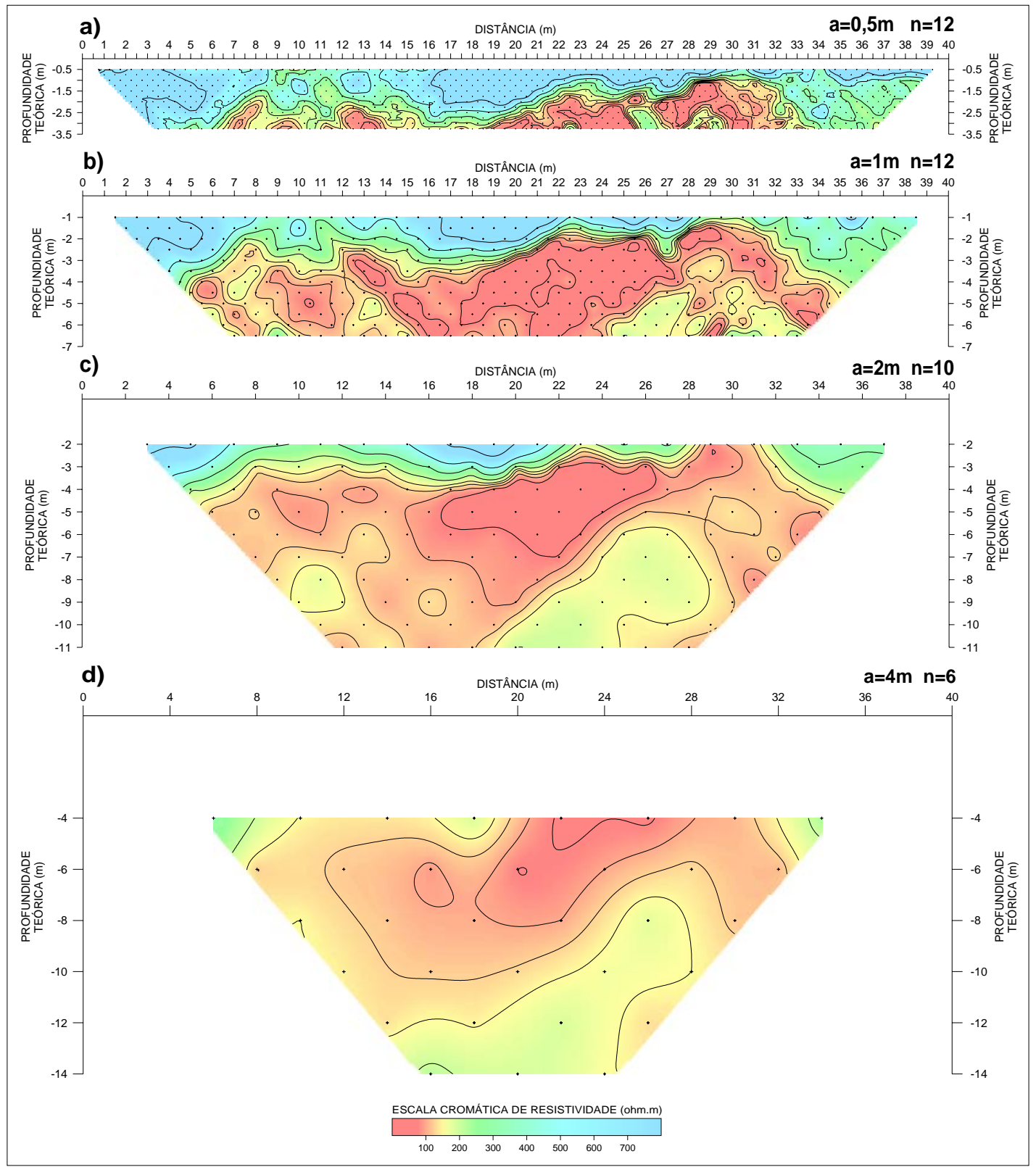

Figura 2 - Pseudo-seções dos caminhamentos com arranjo dipolo-dipolo e espaçamento entre dipolos igual a $0,5 \mathrm{~m}$ (a), $1 \mathrm{~m}$ (b), $2 \mathrm{~m}$ (c) e $4 \mathrm{~m}$ (d).

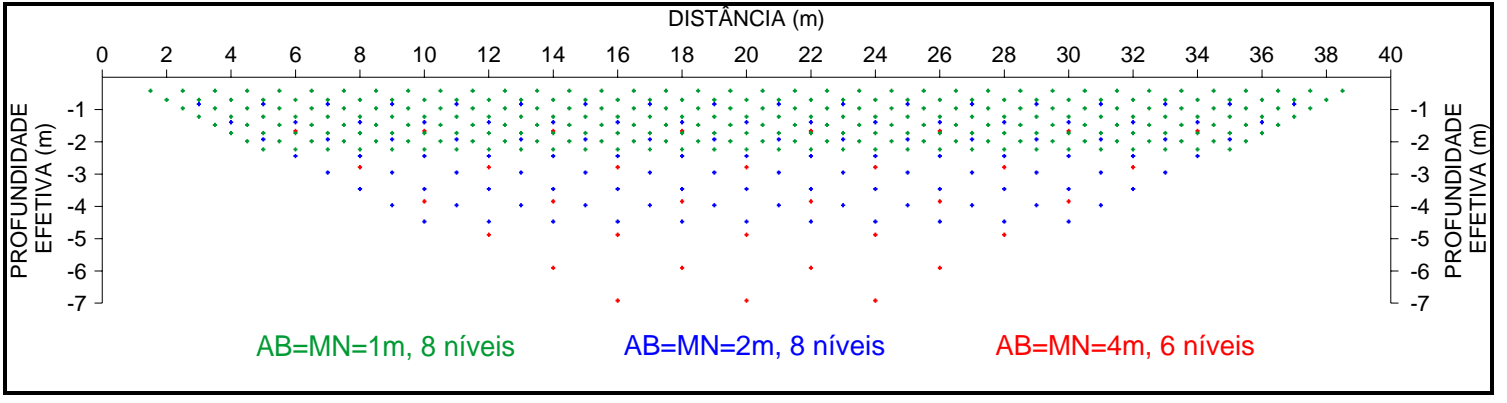

Figura 3 - Plotagem de um conjunto de dados ( $\mathrm{a}=\mathrm{AB}=\mathrm{MN}=4 \mathrm{~m}, 2 \mathrm{~m}$ e $1 \mathrm{~m})$ em uma "pseudo-seção modificada". 


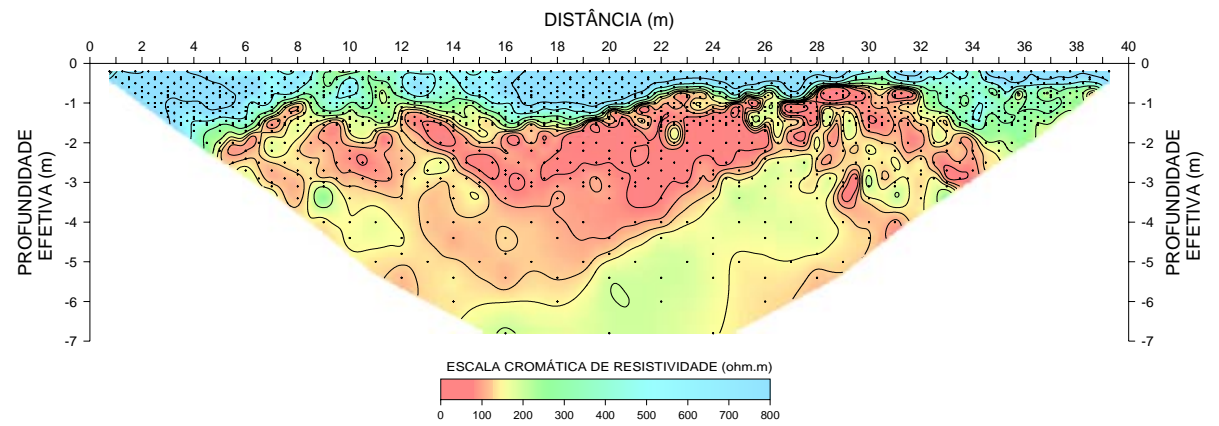

Figura 4 - Pseudo-seção modificada, arranjo dipolo-dipolo, espaçamento entre eletrodos igual a 4m, 2m, 1m e 0,5m.

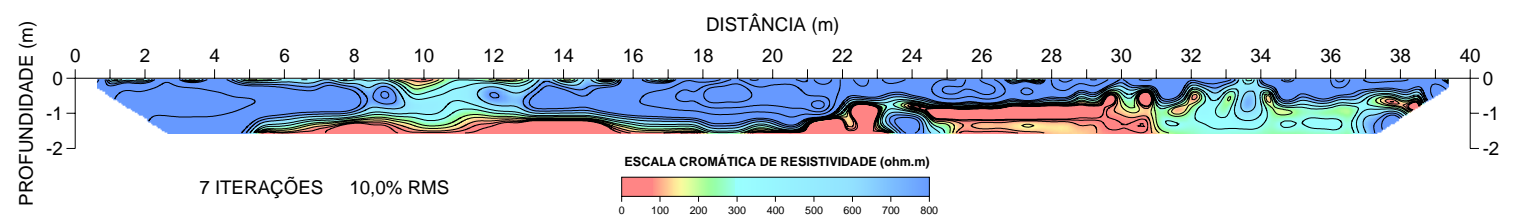

Figura 5 - Seção modelada correspondente à aquisição com espaçamento entre dipolos igual a 0,5m

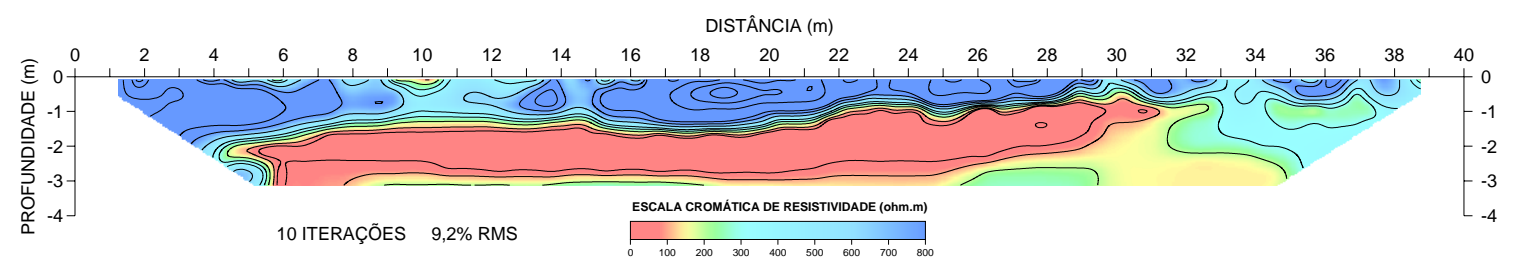

Figura 6 - Seção modelada correspondente à aquisição com espaçamento entre dipolos igual a 1m

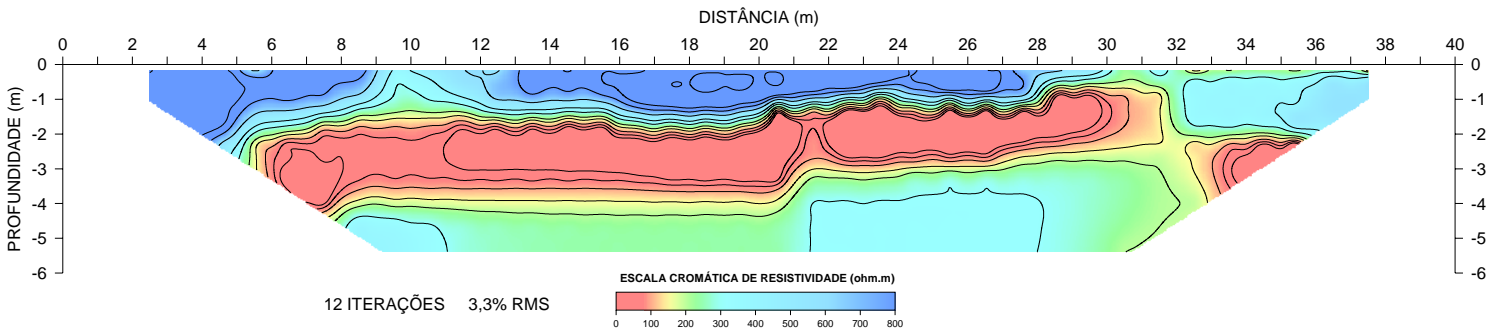

Figura 7 - Seção modelada correspondente à aquisição com espaçamento entre dipolos igual a $2 \mathrm{~m}$

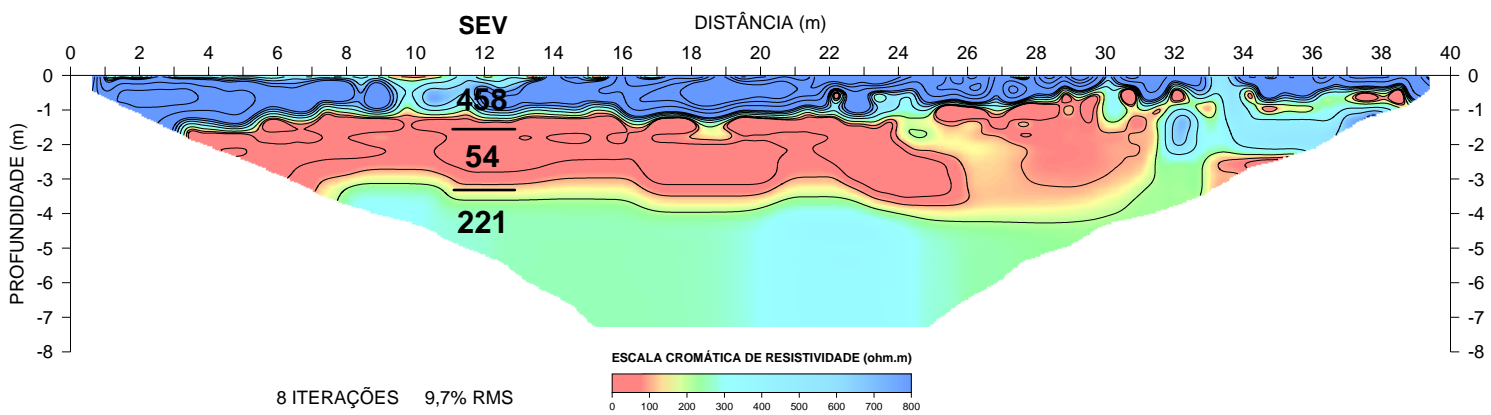

Figura 8 - Seção Final modelada com todos os espaçamentos entre dipolos (0,5m, 1m, 2m e 4m). 